\title{
Distribuição espacial de Aphis gossypii (Glover) (Hemiptera, Aphididae) e Bemisia tabaci (Gennadius) biótipo B (Hemiptera, Aleyrodidae) em algodoeiro Bt e não-Bt
}

\author{
Tatiana Rojas Rodrigues ${ }^{1}$, Marcos Gino Fernandes ${ }^{1}$ \& Honório Roberto dos Santos ${ }^{2}$
}

'Programa de Pós-graduação em Entomologia e Conservação da Biodiversidade, Faculdade de Ciências Biológicas e Ambientais, Universidade Federal da Grande Dourados, Caixa Postal 322, 70804-970 Dourados-MS, Brasil. tathy_r@yahoo.com.br; marcosfernandes@ufgd.edu.br

${ }^{2}$ Programa de Pós-graduação em Entomologia e Conservação da Biodiversidade, Faculdade de Ciências Agrárias, Universidade Federal da Grande Dourados, Caixa Postal 322, 70804-970 Dourados-MS, Brasil. honoriosantos@ufgd.edu.br

\begin{abstract}
Spatial distribution of Aphis gossypii (Glover) (Hemiptera, Aphididae) and Bemisia tabaci (Gennadius) biotype B (Hemiptera, Aleyrodidae) on Bt and non-Bt cotton. The study of spatial distribution of the adults of Bemisia tabaci and the colonies of Aphis gossypii on $B t$ and non-Bt cotton crop is fundamental for improving the sampling techniques and revealing differences in the behavior of non-target species between both cultivars. So, this experiment aimed to investigate the spatial distribution pattern of those species on the conventional non-Bt cotton crop, compared to the $B t$ cultivar. The samplings took place in two areas of 5.000 $\mathrm{m}^{2}$ each, where it was done 14 evaluations counting adults of white-flies and colonies of aphids. The aggregation indexes (variance/ mean rate, index of Morisita and Exponent $\mathrm{k}$ of the Negative Binomial Distribution) were calculated and accomplished the fitness tests of the individuals' numeric classes found and expected to the theoretical distributions of frequency (Poisson, Negative Binomial and Positive Binomial). Those analyses showed that in both cultivars, the spatial distribution of $B$. tabaci fitted to negative binomial distribution throughout the study period, indicating that the transgenic cultivar did not influence the pattern of aggregate distribution of this insect. With respect to the analysis for A. gossypii, the aggregation indexes showed aggregated distribution in both cultivars, but the distributions of frequency pointed to the occurrence of aggregate distribution only on conventional cotton, since there was no adjustment to the data on $B t$ crop. This indicates that $B t$ cotton has altered the aphids normal pattern of dispersion in the crop.
\end{abstract}

\begin{abstract}
KEYWORDS. Cotton-aphid; GMO; Gossypium hirsutum; insecta; white-fly.
RESUMO. Distribuição espacial de Aphis gossypii (Glover) (Hemiptera, Aphididae) e Bemisia tabaci (Gennadius) biótipo B (Hemiptera, Aleyrodidae) em algodoeiro Bt e não-Bt. O estudo da distribuição espacial de adultos de Bemisia tabaci e de Aphis gossypii nas culturas do algodoeiro $B t$ e não- $B t$ é fundamental para a otimização de técnicas de amostragens, além de revelar diferenças de comportamento de espécies não-alvo dessa tecnologia $B t$ entre as duas cultivares. Nesse sentido, o experimento buscou investigar o padrão da distribuição espacial dessas espécies de insetos no algodoeiro convencional não-Bt e no cultivar Bt. As avaliações ocorreram em dois campos de $5.000 \mathrm{~m}^{2}$ cada, nos quais se realizou 14 avaliações com contagem de adultos da moscabranca e colônias de pulgões. Foram calculados os índices de agregação (razão variância/média, índice de Morisita e Expoente k da Distribuição Binomial Negativa) e realizados os testes ajustes das classes numéricas de indivíduos encontradas e esperadas às distribuições teóricas de freqüência (Poisson, Binomial Negativa e Binomial Positiva). Todas as análises mostraram que, em ambas as cultivares, a distribuição espacial de $B$. tabaci ajustou-se a distribuição binomial negativa durante todo o período analisado, indicando que a cultivar transgênica não influenciou o padrão de distribuição agregada desse inseto. Já com relação às análises para A. gossypii, os índices de agregação apontaram distribuição agregada nas duas cultivares, mas as distribuições de freqüência permitiram concluir a ocorrência de distribuição agregada apenas no algodoeiro convencional, pois não houve nenhum ajuste para os dados na cultivar $B t$. Isso indica que o algodão $B t$ alterou o padrão normal de dispersão dos pulgões no cultivo.
\end{abstract}

PALAVRAS-CHAVE. Gossypium hirsutum L; Insecta; mosca-branca; OGM; pulgão-do-algodoeiro.

O pulgão-do-algodoeiro Aphis gossypii (Glover, 1877) (Hemiptera, Aphididae) e a mosca-branca Bemisia tabaci (Gennadius, 1899) Biótipo B (Hemiptera, Aleyrodidae) são importantes vetores de vírus causadores de sérias doenças ao algodoeiro, sobretudo no Brasil. Dentre as viroses transmitidas por esses organismos tem-se o vírus do mosaico-das-nervuras do algodoeiro (VMNA), a doença azul e o mosaico comum (Araújo \& Suassuna 2003; Michelotto \& Busoli 2007). Além da habilidade em transmitir viroses às plantas, altas infestações dessas pragas depauperam as plantas de algodão dada a grande quantidade de assimilados retiradas através do processo de alimentação e ao acúmulo de fumagina devido à deposição de resíduos açucarados nas estruturas das plantas, que contribuem para a queda das folhas e depreciação da pluma, podendo afetar seriamente a produção, como destacado por Santos (2001).

O manejo dessas espécies pragas tem se mostrado um grande desafio, devido principalmente a sua rápida dispersão entre as culturas, o alto potencial reprodutivo, o hábito polífago, a resistência aos inseticidas e seu comportamento de se alimentar e viver na superfície abaxial das folhas (Naranjo \& Flint 1995). Entre as possíveis soluções, em longo prazo, para o controle da mosca-branca e do pulgão-do-algodoeiro, destaca-se o manejo integrado, no qual se utiliza, como tática prioritária, a resistência de cultivares, como enfatiza Chu et al. (1995) e Chu et al. (2001). Nesse sentido, o algodão 
transgênico ' $B t$ ' tem sido inserido como uma alternativa de plantas resistentes a algumas lagartas. Esse algodão é resultante da tecnologia que inseriu alguns genes da bactéria Bacillus thuringiensis no genoma da planta do algodão. Esses genes inseridos são responsáveis pela codificação de uma proteína chamada Cry1Ac que é tóxica para as larvas de algumas espécies de lepidópteros.

Ainda não se sabe, com exatidão, como as plantas geneticamente modificadas afetam as populações de organismos que se inter-relacionam em um agroecossistema. Nesse sentido, o conhecimento da possível alteração na forma da disposição espacial de insetos pragas e/ou benéficos, é extremamente importante, pois pode alterar o método de amostragem dessas espécies na cultura, além da sua forma de controle. Para se determinar o padrão de arranjo espacial de uma determinada espécie em um dado ecossistema é necessário que se tenham dados de contagem de indivíduos (Young \& Young 1998).

Nesse contexto, cabe notar que a distribuição espacial é a forma como os indivíduos de uma população se dispersam em seu habitat (Ricklefs 2003). Seu conhecimento é importante por várias razões: conhecer a etologia da espécie, aperfeiçoar os processos de amostragens e, conseqüentemente, o processo de manejo integrado de pragas agrícolas, entre outras. A forma de dispersão no habitat pode se dar de formas diferentes entre as espécies e entre as populações da mesma espécie; a variação dessa forma de distribuição espacial pode ocorrer em função de fatores ambientais ou genéticos da população. A determinação desses padrões de arranjo é obtida através de índices de dispersão e distribuição teórica de freqüências (Barbosa 1992; Young \& Young 1998; Fernandes et al. 2003).

Segundo Barbosa (1992), para estudos sobre a distribuição de insetos há necessidade de se conhecer as distribuições de freqüências dos indivíduos de cada espécie em cada cultura, adotando-se critérios adequados de amostragem para estimar os parâmetros populacionais. Conforme Kuno (1991), inicialmente deve-se dividir a área para estudo em várias unidades ou quadrados (grids) de mesmo tamanho e, posteriormente, descrever o modelo de ocupação da área pelos indivíduos da população como uma distribuição de freqüências dos indivíduos observados em cada quadrado. $\mathrm{O}$ arranjo espacial de populações de insetos obedece a padrões que podem ser de maneira aleatória, uniforme, ou, ainda, agregada (Ricklefs 2003). A determinação desses padrões de arranjo é obtida através de índices de dispersão e das distribuições teóricas de freqüência (Barbosa 1992).

Cabe enfatizar que o controle de lagartas efetuado pelo algodão $B t$, embora se apresente como uma ferramenta adequada para o manejo de algumas pragas, possibilita o risco de facilitar, indiretamente, um aumento de populações de pragas não-alvo devido à desocupação do nicho ecológico das lagartas, ou devido a efeitos inesperados causados pela transgenia (Liu et al. 2005). É, portanto, possível considerar que a forma como as populações de pulgões e moscas-brancas se distribuem espacialmente no habitat agrícola seja distinta entre culturas transgênicas e não-transgênicas, haja vista que mesmo em variedades "isolinhas" podem haver efeitos bastante diferentes sobre as espécies não-alvos. Portanto, determinar o tipo de distribuição espacial dessas pragas, em ambos os tipos de algodoeiro, é importante para que se possa preparar um programa de amostragem de pragas que busque minimizar o uso de inseticidas químicos tanto na cultivar transgênica, quanto na convencional.

Diante do exposto, conduziu-se a presente pesquisa buscando investigar o padrão da distribuição espacial de $A$. gossypii e $B$. tabaci na cultura do algodoeiro convencional, cultivar DeltaOpal ${ }^{\circledR}$, em comparação com a cultivar transgênica $B t$ DP90 $\mathrm{B}^{\circledR}$.

\section{MATERIAL E MÉTODOS}

\section{Descrição da área amostral}

Os experimentos foram conduzidos durante a safra agrícola 2006/2007, em área agrícola localizada no município de Caarapó, região sul do Estado de Mato Grosso do Sul. A área utilizada era de 300 ha, irrigados com pivô central, na qual foi realizada a seguinte distribuição das cultivares para a semeadura: $80 \%$ da cultivar DP90B ${ }^{\circledR}(B t)$ e $20 \%$ com a cultivar de algodão DeltaOpal ${ }^{\circledR}$ (convencional). O tratamento de sementes foi realizado com Euparen ${ }^{\circledR}+$ Monaron $^{\circledR}+$ Baytan $^{\circledR}$ (fungicidas) e Cruiser ${ }^{\circledR} 700 \mathrm{WS}$ (inseticida) e a emergência das plantas ocorreu no dia 13 de dezembro para o algodão $B t$ e no dia 27 de dezembro de 2006 para o convencional.

Quanto às coordenadas geográficas da área amostral temse: latitude de $22^{\circ} 30^{\prime} \mathrm{S}$ e longitude de $54^{\circ} 49^{\prime} \mathrm{W}$. A temperatura média, desde o $1^{\circ}$ dia após a emergência das plantas (DAE) até a última avaliação, foi de $26,8^{\circ} \mathrm{C}$, sendo a temperatura mínima de $14,6^{\circ} \mathrm{C}$, no dia $19 / 03 / 2007$, e a máxima de $36,1^{\circ} \mathrm{C}$, no dia $16 / 03 / 2007$. A média de precipitação de água da chuva foi de $6,7 \mathrm{~mm}$, de 13 de dezembro a 25 de março, o que compreendeu o período das avaliações; e a maior chuva ocorreu no dia 06/02/2007 totalizando $66 \mathrm{~mm}$. O solo predominante da região é o Latossolo Vermelho Distroférrico que se apresenta com textura argilosa e fertilidade natural variável, além de textura média e caráter álico, porém, é profundo, friável e com grande homogeneidade ao longo de todo o perfil. Em relação ao relevo é plano e suavemente ondulado apresentando cobertura vegetal natural de Floresta Estacional Semidecidual e região de Cerrado.

\section{Amostragens}

As amostragens foram realizadas da seguinte forma: na área amostral foram demarcados dois campos de $5.000 \mathrm{~m}^{2}$, sendo cada um deles dividido em 100 parcelas de $50 \mathrm{~m}^{2}(5$ $\mathrm{m} \times 10 \mathrm{~m}$ ). Em cada parcela foram avaliadas duas plantas em seqüência, totalizando 200 plantas em cada campo. As amostragens consistiam de contagem de indivíduos adultos de B. tabaci e colônias de A. gossypii na planta inteira. Devido à facilidade com que os adultos de mosca-branca voam quando as plantas são agitadas, fez-se observação cuidadosa a partir do ponteiro até as folhas mais baixas de cada planta, manuseando-se as folhas muito lentamente. Considerou-se uma colônia como o grupo de, no mínimo, sete indivíduos (pulgões ápteros ou alados) e a cada observação era anotado o número de colônias destes insetos presentes em cada planta. 
Tabela I. Estatísticas \{médias ( $\hat{m}$ ) e variâncias ${ }_{(\mathrm{s}}^{2}$ ) de colônias de Aphis gossypii e de indivíduos de Bemisia tabaci Biótipo B por unidade amostral\} e índices de dispersão \{razão variância/média $(\boldsymbol{I})$; índice de Morisita $\left(\boldsymbol{I}_{\hat{\delta}}\right)$ e expoente $\mathrm{K}$ da binomial negativa $(\mathbf{K})$ \} em algodoeiro geneticamente modificado cultivar DP90B ${ }^{\circledR}$, Caarapó, MS, 2007.

\begin{tabular}{|c|c|c|c|c|c|c|c|c|c|c|c|c|c|c|c|}
\hline & & $3 \mathrm{DAE}$ & DAE & $2 \mathrm{DAE}$ & 49DAE & $\mathrm{EE}$ & $9 \mathrm{DAE}$ & $63 \mathrm{DAE}$ & $73 \mathrm{DAE}$ & $77 \mathrm{DAE}$ & 80DAE & $84 \mathrm{DAE}$ & 88DAE & $95 \mathrm{DAE}$ & $102 \mathrm{DAE}$ \\
\hline \multirow{5}{*}{ 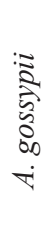 } & $\hat{m}$ & 0 & 0,45 & 0,66 & 1,13 & 0,5 & 0,15 & 0 & 0,06 & 0,08 & 0,3 & 0,1 & 0,51 & 0,13 & 1,64 \\
\hline & $s^{2}$ & - & 1,2803 & 3,4185 & 3,1849 & 1,505 & 0,25 & - & 0,0569 & 0,2965 & 0,3939 & & & & 3,4044 \\
\hline & $I$ & - & $2,8451^{* *}$ & $5,1796^{* *}$ & $2,8185^{* *}$ & $3,0101^{* *}$ & $1,6666^{* *}$ & - & $0,9494^{* *}$ & $3,7070^{* *}$ & $1,3131^{* *}$ & $1,5151^{* *}$ & $2,2774^{* *}$ & $2,5881^{* *}$ & $2,0758^{* *}$ \\
\hline & $I_{\delta}$ & - & $5,1515^{* *}$ & $7,3659^{* *}$ & $2,6074^{* *}$ & $5,0612^{* *}$ & $5,7142^{* *}$ & - & $0^{\text {ns }}$ & $39,2851^{* *}$ & $2,0689^{* *}$ & $6,6666^{* *}$ & & $14,1026^{* *}$ & $1,6534^{* *}$ \\
\hline & $\mathrm{K}$ & - & $0,2438^{\mathrm{ag}}$ & $0,1579^{\mathrm{ag}}$ & $0,6213^{\mathrm{ag}}$ & $0,2487^{\mathrm{ag}}$ & $0,2250^{\mathrm{ag}}$ & - & $-1,188^{\text {al }}$ & $0,0295^{\mathrm{ag}}$ & $0,9580^{\mathrm{ag}}$ & $0,1941^{\mathrm{ag}}$ & 0,39 & 0,08 & $1,5243^{\mathrm{ag}}$ \\
\hline \multirow{5}{*}{ 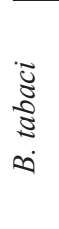 } & $\hat{m}$ & 0,87 & 0,38 & 0,68 & 3,49 & 10,75 & 4,33 & 2,02 & 356 & 003 & 05 & & & & 14 \\
\hline & $\mathrm{s}^{2}$ & 2,0536 & 0,6824 & 1,8763 & 23,5049 & 156,614 & & & 10,85 & 4,4495 & & & & & 12,0064 \\
\hline & $I$ & $2,3605^{* *}$ & $1,7958^{* *}$ & $2,7593^{* *}$ & $6,7349^{* *}$ & $14,5687^{* *}$ & $3,3828^{* *}$ & $5,2801^{* *}$ & $3,0491^{* *}$ & $4,7845^{* *}$ & $2,3352^{* *}$ & $1,7676^{* *}$ & $1,9345^{* *}$ & $2,1105^{* *}$ & $2,7041^{* *}$ \\
\hline & $I_{\delta}$ & $2,5661^{* *}$ & $3,1294^{* *}$ & $3,5996^{* *}$ & $2,6314^{* *}$ & $2,2507^{* *}$ & $1,5460^{* *}$ & $2,6234^{* *}$ & $1,5714^{* *}$ & $5,0724^{* *}$ & $3,4940^{* *}$ & $2,7272^{* *}$ & $4,5584^{* *}$ & $4,0540^{* *}$ & $1,3808^{* *}$ \\
\hline & $\mathrm{K}$ & $0,6394^{\mathrm{ag}}$ & $0,4774^{\mathrm{ag}}$ & $0,3865^{\mathrm{ag}}$ & $0,6085^{\mathrm{ag}}$ & $0,7922^{\mathrm{ag}}$ & $1,8171^{\mathrm{ag}}$ & $0,6121^{\mathrm{ag}}$ & $1,7373^{\mathrm{ag}}$ & $0,2457^{\mathrm{ag}}$ & $0,4044^{\mathrm{ag}}$ & $0,5861^{\mathrm{ag}}$ & $0,2889^{\mathrm{ag}}$ & $0,3331^{\mathrm{ag}}$ & $2,6053^{\mathrm{ag}}$ \\
\hline
\end{tabular}

$\mathrm{DAE}=$ dias após a emergência das plantas; $; *$ * = significativo no nível de $1 \%$ pelo teste do qui-quadrado; ${ }^{\text {ag }}=$ agregada; ${ }^{\text {al }}=$ aleatório

As avaliações, em ambas as cultivares, foram realizadas semanalmente até o surgimento da primeira flor e após o aparecimento do primeiro capulho, sendo que, entre o surgimento da primeira flor e do primeiro capulho as amostragens foram realizadas duas vezes por semana. As amostragens das colônias de $A$. gossypii nas áreas amostrais iniciaram-se no dia 10/01/2007, correspondente ao $15^{\circ}$ DAE (dia após a emergência), na cultivar convencional, e ao $28^{\circ}$ DAE, na cultivar Bt. Já a primeira avaliação da população de B. tabaci foi realizada no $15^{\circ} \mathrm{DAE}$, na cultivar convencional, e no $28^{\circ} \mathrm{DAE}$, na cultivar $B t$. O período total de amostragem foi de 10/01/07 à 25/03/07, totalizando 14 avaliações em cada cultivar. Não foram realizadas aplicações de inseticidas no período que ocorreram as avaliações.

As análises da estatística ecológica, realizadas para se determinar o padrão de distribuição espacial, utilizaram as médias do número de indivíduos encontrados em cada área com total de 100 parcelas e basearam-se na utilização dos seguintes parâmetros:

\section{Índices de agregação}

Razão Variância/Média: esta razão $(I)$ é um índice que serve para medir o desvio de um arranjo dos dados da aleatoriedade. Para este índice os valores iguais à unidade indicam disposição espacial ao acaso ou aleatória; já os valores menores indicam que a unidade aponta disposição espacial regular ou uniforme, e os valores significativamente maiores que 1 mostram disposição agregada ou contagiosa (Rabinovich 1980).

Índice de Morisita: o índice de Morisita $\left(I_{\delta}\right)$ é relativamente independente da média e do número de amostras. Sendo assim, quando $I_{\delta}=1$ a distribuição é ao acaso; quando $I_{\delta}>1 \mathrm{a}$ distribuição é do tipo contagiosa e quando $I_{\delta}<1$ indica uma distribuição regular (Silveira Neto et al. 1976).

Expoente k da Distribuição Binomial Negativa: o expoente $\mathrm{k}$ é um índice adequado de dispersão quando o tamanho e os números de unidades amostrais são os mesmos em cada amostra, já que, freqüentemente, este é influenciado pelo tamanho das unidades amostrais. Este parâmetro é uma medida inversa do grau de agregação, nesse caso os valores negativos indicam uma distribuição regular ou uniforme, os valores positivos, próximos de zero, indicam disposição agregada e os valores superiores a oito indicam uma disposição ao acaso (Southwood 1978; Elliot 1979). Sobre esse aspecto, Poole (1974) utiliza outra interpretação, para ele quando $0<\mathrm{k}<8$, o índice indica distribuição agregada, e quando $0>\mathrm{k}>8$ aponta para distribuição aleatória.

Distribuição teórica de freqüências

As distribuições teóricas de freqüências utilizadas para avaliar a distribuição espacial das espécies observadas são apresentadas a seguir, de acordo com Young \& Young (1998).

Distribuição de Poisson: também conhecida como distribuição aleatória, caracteriza-se por apresentar variância igual à média $\left(\mathrm{s}^{2}=\hat{m}\right)$.

Distribuição Binomial Positiva: descreve a distribuição uniforme e apresenta variância menor que a média $\left(\mathrm{s}^{2}<\mathrm{m}\right)$.

Distribuição Binomial Negativa: apresenta variância maior que a média, indicando, assim, distribuição agregada, além de possuir dois parâmetros: a média $(\mathrm{m})$ e o parâmetro $\mathrm{k}(\mathrm{k}>0)$.

Teste de qui-quadrado de aderência: para a verificação do teste de ajuste dos dados coletados em campo às distribuições teóricas de freqüência, utilizou-se o teste qui-quadrado de aderência que compara o total das freqüências observadas na área amostral, com as freqüências esperadas, de acordo com Young \& Young (1998); sendo estas freqüências definidas pelo produto das probabilidades de cada classe e o número total de unidades amostrais utilizadas. Para a realização deste teste, optou-se por fixar uma freqüência esperada mínima igual à unidade. 
Tabela II. Estatísticas \{médias $(\hat{m})$ e variâncias $\left({ }_{\mathrm{s}}{ }^{2}\right)$ de colônias de Aphis gossypii e de indivíduos de Bemisia tabaci Biótipo B por unidade amostral\} e índices de dispersão \{razão variância/média (I); índice de Morisita $\left(\boldsymbol{I}_{\delta}\right)$ e expoente $\mathrm{K}$ da binomial negativa $(\mathbf{K})$ \} em algodoeiro cultivar DeltaOpal ${ }^{\circledR}$, Caarapó, MS, 2007.

\begin{tabular}{|c|c|c|c|c|c|c|c|c|c|c|c|c|c|c|c|}
\hline & & & & & & & & & & & & & & & \\
\hline \multirow{5}{*}{ 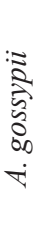 } & $\hat{m}$ & 0,04 & 1,49 & & 26 & & & & & & & (2) & & 06 & 1,58 \\
\hline & $s^{2}$ & 0,16 & 4,4746 & 4,9546 & & 0,1691 & & & & & & & & 1,2727 & 3,2965 \\
\hline & $I$ & $4^{* *}$ & $3,0031^{* *}$ & $3,1558^{* *}$ & & 年 & $3063^{* * *}$ & & & & $2,6709^{* *}$ & $2,0981^{* *}$ & & & \\
\hline & $I_{\delta}$ & $100^{* *}$ & $2,3399^{* *}$ & $2,3681^{* *}$ & & $60^{* *}$ & $46,6666^{* *}$ & $6^{* *}$ & & 6,66 & $14^{* *}$ & & & 2,88 & \\
\hline & K & $0,0133^{\mathrm{ag}}$ & $0,7438^{\mathrm{ag}}$ & $0,7282^{\mathrm{ag}}$ & $2,6205^{\mathrm{ag}}$ & $0209^{\text {ag }}$ & $0,0260^{\mathrm{ag}}$ & & $0,0304^{\mathrm{ag}}$ & $1871^{\mathrm{ag}}$ & $0,0418^{\mathrm{ag}}$ & $0,2549^{\mathrm{ag}}$ & & 0,53 & $1,4542^{\mathrm{ag}}$ \\
\hline \multirow{5}{*}{ 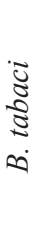 } & $\hat{m}$ & 0,48 & & & & & & & & & & & & & \\
\hline & $s^{2}$ & 0,8985 & 0,7519 & 0,808 & & 0,0771 & & & & & & & & & \\
\hline & $I$ & $1,8720^{* *}$ & $2,2115^{* *}$ & $2,0202^{* *}$ & $5,1184^{* *}$ & $1,2861^{* *}$ & & & & & & & & & $1,8871^{* *}$ \\
\hline & $I_{\delta}$ & $2,8368^{* *}$ & $4,6345^{* *}$ & $3,5897^{* *}$ & $7,2727^{* *}$ & $6,6666^{* *}$ & $2,1960^{* *}$ & & & & & $2,5974^{* *}$ & & $2,2739^{* *}$ & $2,5408^{* *}$ \\
\hline & K & $0,5504^{\mathrm{ag}}$ & $0,2806^{\mathrm{ag}}$ & $0,3920^{\mathrm{ag}}$ & $0,1602^{\mathrm{ag}}$ & $0,2096^{\mathrm{ag}}$ & $0,8442^{\mathrm{ag}}$ & $0,7095^{\mathrm{ag}}$ & $0,1783^{\mathrm{ag}}$ & $0,1267^{\mathrm{ag}}$ & $0,6724^{\mathrm{ag}}$ & $0,6310^{\mathrm{ag}}$ & $-1,98^{\text {al }}$ & $0,7898^{\mathrm{ag}}$ & $0,6537^{\mathrm{ag}}$ \\
\hline
\end{tabular}

$\mathrm{DAE}=$ dias após a emergência das plantas;; **= significativo no nível de $1 \%$ pelo teste do qui-quadrado; ${ }^{\text {ag }}=$ agregada; ${ }^{\text {al }}=$ aleatório

\section{RESULTADOS E DISCUSSÃO}

\section{Colônias de A. gossypii}

Foi encontrado maior número de colônias de pulgões na cultivar $B t$ do que na não- $B t$ (Tabela I e Tabela II). Essa maior infestação na cultivar $B t$ deveu-se, provavelmente, à diminuição de competição inter-específica entre as diversas populações que compunham a comunidade de artrópodes da cultura, isso foi ocasionado, provavelmente, devido à redução da população de lagartas de Alabama argillacea (Hubner, 1818), Heliothis virescens (Fabricius, 1781) (Lepidoptera, Noctuidae) e Pectinophora gossypiella (Saunders, 1843) (Lepidoptera, Gelechiidae), espécies essas que são alvo da toxina Cry1Ac e que, portanto, não foram encontradas nessa cultivar em virtude da resistência oferecida por essas plantas transgênicas a esses lepidópteros. Em todas as avaliações na cultivar convencional foram encontradas colônias deste afídeo; já na cultivar $B t$, em algumas amostragens (que correspondem aos 28 e 63 DAE) não foi encontrada nenhuma colônia.

Índices de Agregação: A razão variância/média (I) calculada para $A$. gossypii exibiu valores maiores que a unidade em todas as avaliações realizadas na cultivar convencional, com os valores variando de 1,8013 a 4,1519 (Tabela II). Esta situação não ocorreu com a cultivar Bt, pois, dentre as 14 avaliações realizadas nessa cultivar, aos $28 \mathrm{DAE}$ e 63 DAE não foram encontradas colônias, enquanto que na amostragem realizada aos 73 DAE a relação variância/ média foi de 0,9494 , ou seja, menor que a unidade, indicando uniformidade.

Pelos resultados obtidos com os índices de Morisita, verifica-se que em todas as amostragens, e em ambas as variedades (Tabelas I e II), os valores foram superiores à unidade com significância de $1 \%$ de probabilidade, excetuando-se a primeira (28 DAE) e a sétima (63 DAE) avaliação na cultivar $B t$, nas quais não houve ocorrência de colônias deste hemíptero, e a oitava (73DAE) avaliação, também nesta cultivar, quando a média de indivíduos foi muito baixa e o índice de Morisita resultou em 0,06.

Analisando-se o expoente $\mathrm{K}$ verificou-se que, em todas as avaliações realizadas na cultivar convencional, o arranjo espacial das colônias tenderam à agregação, pois os valores desse índice variaram de 0,0295 até 1,4542 (Tabela II); já com relação à cultivar $B t$, exceto as $1^{\mathrm{a}}(28 \mathrm{DAE}), 7^{\mathrm{a}}(63 \mathrm{DAE})$ e $8^{a}$ (73 DAE) avaliações, todas as demais também indicaram distribuição do tipo contagiosa (Tabela I).

Após serem calculados e analisados os índices de dispersão, é possível afirmar que todos esses índices indicam que a disposição espacial das colônias de pulgões é de forma agregada em ambas as cultivares estudadas.

Distribuições Teóricas de Freqüências: Os testes de ajustes das freqüências de classes numéricas das colônias de A. gossypii observadas em campo às freqüências calculadas das classes teóricas da Distribuição de Poisson (Tabela III) indicaram que os dados encontrados não obtiveram um bom ajuste ao padrão de distribuição espacial tipo aleatória. Nesse sentido, nota-se que das 14 avaliações realizadas em cada área amostral, apenas sete delas na cultivar convencional $\left\{2^{\mathrm{a}}\right.$ (aos $22 \mathrm{DAE}), 3^{\mathrm{a}}$ (29 DAE), $4^{\mathrm{a}}$ (36 DAE), $9^{\mathrm{a}}(67 \mathrm{DAE}), 11^{\mathrm{a}}$ (75 DAE), $13^{\mathrm{a}}$ (85 DAE) e $14^{\mathrm{a}}$ (89 DAE) $\}$ e nove na cultivar $B t\left\{2^{\mathrm{a}}\right.$ (aos $\left.35 \mathrm{DAE}\right), 3^{\mathrm{a}}(42 \mathrm{DAE}), 4^{\mathrm{a}}(49 \mathrm{DAE}), 5^{\mathrm{a}}(52 \mathrm{DAE})$, $6^{\mathrm{a}}$ (59 DAE), $10^{\mathrm{a}}$ (80 DAE), $12^{\mathrm{a}}$ (88 DAE), $13^{\mathrm{a}}$ (95 DAE) e $14^{\mathrm{a}}$ (102 DAE)\} apresentaram número de classes suficientes para aplicação do teste de qui-quadrado e para ajuste das classes observadas em campo à distribuição teórica de Poisson. Dentre as avaliações que apresentaram suficiência de classes, na cultivar convencional apenas a $9^{\text {a }}$ (67 DAE) amostragem resultou em ajuste, enquanto na cultivar $B t$ a $6^{\mathrm{a}}(59 \mathrm{DAE})$ e a $13^{\mathrm{a}}$ (95 DAE) amostragem mostraram-se ajustadas a esta distribuição; porém, essas três avaliações, mesmo ajustando-se à distribuição de Poisson, resultaram em médias muito baixas de indivíduos encontrados, não permitindo confiabilidade neste resultado. Por outro lado, as $2^{\mathrm{a}}$ (22 DAE), $3^{\mathrm{a}}$ (29DAE), $4^{\mathrm{a}}$ (36 DAE), 13 ${ }^{\mathrm{a}}$ (85DAE) e $14^{\mathrm{a}}$ (89 DAE) amostragens na cultivar convencional, e as $2^{\mathrm{a}}$ (35 DAE), $3^{\mathrm{a}}$ (42 DAE), $4^{\mathrm{a}}$ (49 DAE), $5^{\mathrm{a}}$ (52 DAE), $12^{\mathrm{a}}$ (88 
Tabela III. Teste qui-quadrado de aderência de Aphis gossypii e Bemisia tabaci Biótipo B (Poisson) em algodoeiro DeltaOpal ${ }^{\circledR}$ e DP90B ${ }^{\circledR}$ em Caarapó, MS, 2007.

\begin{tabular}{|c|c|c|c|c|c|c|c|c|}
\hline \multirow[b]{3}{*}{ Amostras } & \multicolumn{4}{|c|}{ DeltaOpal $^{\circledR}$} & \multicolumn{4}{|c|}{ DP90B ${ }^{\circledR}$} \\
\hline & \multicolumn{2}{|c|}{ Aphis gossypii } & \multicolumn{2}{|c|}{ Bemisia tabaci } & \multicolumn{2}{|c|}{ Aphis gossypii } & \multicolumn{2}{|c|}{ Bemisia tabaci } \\
\hline & $\mathrm{X}^{2}$ & GL(nc-2) & $\mathrm{X}^{2}$ & GL(nc-2) & $\mathrm{X}^{2}$ & GL(nc-2) & $\mathrm{X}^{2}$ & GL(nc-2) \\
\hline $1^{\mathrm{a}}$ & $2,26^{\mathrm{i}}$ & 0 & $13,78^{* *}$ & 2 & 0 & - & $38,68^{* *}$ & 3 \\
\hline $2^{\mathrm{a}}$ & $163,88^{* *}$ & 4 & $18,74^{* *}$ & 4 & $35,79^{* *}$ & 2 & $5,69^{*}$ & 1 \\
\hline $3^{\mathrm{a}}$ & $124,12^{* *}$ & 4 & $17,89^{* *}$ & 4 & $39,58^{* *}$ & 2 & $28,24^{* *}$ & 2 \\
\hline $4^{\mathrm{a}}$ & $2124,72^{* *}$ & 15 & $75,19^{* *}$ & 2 & $64,86^{* *}$ & 3 & $595,71^{* *}$ & 7 \\
\hline $5^{\mathrm{a}}$ & $1,78^{\mathrm{i}}$ & 0 & $0,12^{\mathrm{i}}$ & 0 & $20,89^{* *}$ & 3 & $12872,27^{* *}$ & 18 \\
\hline $6^{\mathrm{a}}$ & $2,66^{\mathrm{i}}$ & 0 & $13,02^{* *}$ & 2 & $2,24^{\mathrm{ns}}$ & 1 & $228,91^{* *}$ & 9 \\
\hline $7^{\mathrm{a}}$ & $2,66^{\mathrm{i}}$ & 0 & $59,42^{* *}$ & 3 & - & - & $389,15^{* *}$ & 6 \\
\hline $8^{\mathrm{a}}$ & $1,45^{\mathrm{i}}$ & 0 & $18,82^{* *}$ & 1 & $0,02^{\mathrm{i}}$ & 0 & $220,46^{* *}$ & 8 \\
\hline $9^{\mathrm{a}}$ & $2,23^{\mathrm{ns}}$ & 1 & $21,58^{* *}$ & 2 & $18,03^{\mathrm{i}}$ & 0 & $161,44^{* *}$ & 3 \\
\hline $10^{\mathrm{a}}$ & $1,21^{\mathrm{i}}$ & 0 & $4,63^{*}$ & 1 & $5,58^{*}$ & 1 & $41,87^{* *}$ & 2 \\
\hline $11^{\mathrm{a}}$ & $5,62^{*}$ & 1 & $9,44^{* *}$ & 2 & $3,24^{\mathrm{i}}$ & 0 & $11,39^{* *}$ & 2 \\
\hline $12^{\mathrm{a}}$ & $1,1^{\mathrm{i}}$ & 0 & 0 & 0 & $236,88^{* *}$ & 2 & $22,95^{* *}$ & 1 \\
\hline $13^{\mathrm{a}}$ & $22,98^{* *}$ & 2 & $3,94^{*}$ & 2 & $1,64^{\mathrm{ns}}$ & 2 & $20,02^{* *}$ & 1 \\
\hline $14^{\mathrm{a}}$ & $47,66^{* *}$ & 4 & $15,74^{* *}$ & 2 & $32,59^{* *}$ & 6 & $92,82^{* *}$ & 9 \\
\hline
\end{tabular}

ns - Não significativo, $*$ - significativo ao nível de $5 \%, * *$ - significativo ao nível de $1 \%,{ }^{\mathrm{i}}=$ insuficiência de classes, $\mathrm{X}^{2}$ - valor do qui-quadrado calculado, $\mathrm{GL}$ - graus de liberdade, nc - número de classes observadas no campo.

DAE) e $14^{\mathrm{a}}$ (102 DAE) amostragens na cultivar transgênica, apresentaram significância no nível de $1 \%$ de probabilidade a essa distribuição teórica; apenas a $11^{\mathrm{a}}$ (75 DAE) amostragem na convencional e a $10^{\mathrm{a}}$ (80 DAE) na transgênica apresentaram significância no nível de $5 \%$ de probabilidade. A determinação desse fato permite concluir que houve forte rejeição da disposição espacial das colônias de $A$. gossypii observada no campo à distribuição aleatória.

Os testes de ajuste das classes numéricas à distribuição binomial negativa (Tabela IV) permitem concluir que estes insetos apresentam uma forma de distribuição com ajuste muito bom a este tipo de distribuição teórica na cultivar $B t$; pois, dentre as sete amostragens $\left\{2^{\mathrm{a}}\right.$ (35 DAE), $3^{\mathrm{a}}$ (42 DAE), $4^{\mathrm{a}}$ (49 DAE), $5^{\mathrm{a}}$ (52 DAE), 12 $2^{\mathrm{a}}$ (88 DAE), 13 ${ }^{\mathrm{a}}$ (95 DAE) e $14^{\mathrm{a}}$ (102 DAE)\} com número de classes suficientes para realização do teste de ajuste, apenas na $12^{\text {a }}$ não se observou perfeito ajuste a este tipo de distribuição. Essa disposição revela que o arranjo espacial das colônias de $A$. gossypii na cultivar transgênica pode ser considerado como fortemente agregada. Por outro lado, com relação a cultivar não transgênica, houve sete amostragens $\left\{\left(2^{\mathrm{a}}\right.\right.$ (22 DAE), $3^{\mathrm{a}}(29$ DAE $), 4^{\mathrm{a}}$ (36 DAE), $11^{\mathrm{a}}$ (75 DAE), $13^{\mathrm{a}}$ (85 DAE) e $14^{\mathrm{a}}(89$ DAE) \} com número de classes aceitável para a realização do teste, mas apenas a $13^{\mathrm{a}}$ amostragem ajustou-se a distribuição binomial negativa apresentando não significância no teste de qui-quadrado, indicando agregação; já as $2^{\mathrm{a}}$ e $4^{\mathrm{a}}$ amostragens revelaram significância com $1 \%$ de probabilidade, enquanto nas $3^{\mathrm{a}}, 11^{\mathrm{a}}$ e $14^{\mathrm{a}}$ os ajustes ocorreram com significância no nível de $5 \%$ de probabilidade.

Cabe enfatizar, também, que não foi possível realizar, adequadamente, o teste de qui-quadrado de aderência das classes numéricas encontradas em campo à distribuição binomial positiva (Tabela $\mathrm{V}$ ), pois dentre todas as amostragens realizadas nas cultivares $B t$ e não- $B t$, nenhuma obteve número de classes suficientes para o teste de ajustes a essa distribuição teórica. Conclui-se, assim, que as colônias de indivíduos desse afídeo não apresentam nenhuma tendência para esse tipo de distribuição.

Portanto, após o ajuste das freqüências de classes numéricas observadas em campo aos três tipos de distribuições teóricas de freqüência, é possível afirmar que a forma de arranjo espacial horizontal das colônias de $A$. gossypii, em ambas as cultivares avaliadas, é agregada, situação que segundo Ricklefs (2003), pode ser resultante de tendências sociais dos indivíduos em formarem grupos com diferentes finalidades como: segurança, acasalamento ou reprodução. É, realmente, esperado que a disposição das colônias de A. gossypii em área de algodão ocorra em grupos (ou em reboleiras), principalmente, no período inicial da infestação, tendo em vista que a colonização se inicia a partir de alados migrantes. Com o passar do tempo, caso a população aumente muito, a distribuição das colônias tende a tornar-se regular em todo o campo (Degrande 1998).

\section{Adultos de B. tabaci}

Índices de agregação: Após a confecção dos cálculos do expoente $\mathrm{K}$ da binomial negativa, verificou-se que, em praticamente todas as avaliações realizadas, os adultos de $B$. tabaci tenderam a disposição agregada em ambas as cultivares. Na cultivar convencional, de um total de 14 amostragens realizadas, 13 apresentaram valores desse índice variando entre 0,1267 e 0,8442 , exceto aos 82 DAE quando o índice foi de $-1,98$ indicando aleatoriedade (Tabela II); já na cultivar $B t$ os índices apresentaram-se entre 0,2457 e 2,6053, também indicando disposição agregada (Tabela I).

Ao se considerar a relação variância/média, em quase todas as amostragens realizadas observou-se valores maiores que a unidade em ambas cultivares. Esses valores variaram de 1,2861 a 5,1184 na cultivar convencional, descrevendo a distribuição agregada; uma única exceção ocorreu aos 82 
Tabela IV. Teste qui-quadrado de aderência de Aphis gossypii e Bemisia tabaci Biótipo B (Binomial Negativa) em algodoeiro DeltaOpal ${ }^{\circledR}$ e DP90B ${ }^{\circledR}$ em $^{-1}$ Caarapó, MS, 2007.

\begin{tabular}{|c|c|c|c|c|c|c|c|c|}
\hline \multirow[b]{3}{*}{ Amostras } & \multicolumn{4}{|c|}{ DeltaOpal $^{\circledR}$} & \multicolumn{4}{|c|}{ DP90B $^{\circledR}$} \\
\hline & \multicolumn{2}{|c|}{ Aphis gossypii } & \multicolumn{2}{|c|}{ Bemisia tabaci } & \multicolumn{2}{|c|}{ Aphis gossypii } & \multicolumn{2}{|c|}{ Bemisia tabaci } \\
\hline & $\mathrm{X}^{2}$ & GL(nc-3) & $\mathrm{X}^{2}$ & GL(nc-3) & $\mathrm{X}^{2}$ & GL(nc-3) & $\mathrm{X}^{2}$ & GL(nc-3) \\
\hline $1^{\mathrm{a}}$ & 0,26 & - & $0,37^{\mathrm{ns}}$ & 2 & 0 & - & $3,55^{\mathrm{ns}}$ & 4 \\
\hline $2^{\mathrm{a}}$ & $23,11^{* *}$ & 5 & $4,13^{\mathrm{ns}}$ & 2 & $7,65^{\mathrm{ns}}$ & 3 & $0,97^{\mathrm{ns}}$ & 1 \\
\hline $3^{\mathrm{a}}$ & $15,72^{*}$ & 6 & $5,31^{\mathrm{ns}}$ & 2 & $10,24^{\mathrm{ns}}$ & 6 & $3,95^{\text {ns }}$ & 4 \\
\hline $4^{\mathrm{a}}$ & $43,07^{* *}$ & 18 & $7,87^{\mathrm{ns}}$ & 4 & $4,84^{\mathrm{ns}}$ & 5 & $34,13^{* *}$ & 12 \\
\hline $5^{\mathrm{a}}$ & 0,05 & - & 0 & - & $2,53^{\mathrm{ns}}$ & 3 & $47,41^{* *}$ & 22 \\
\hline $6^{\mathrm{a}}$ & $2,55^{\mathrm{i}}$ & 0 & $0,66^{\mathrm{ns}}$ & 1 & $0,24^{\mathrm{i}}$ & 0 & $19,95^{\mathrm{ns}}$ & 15 \\
\hline $7^{\mathrm{a}}$ & $2,55^{\mathrm{i}}$ & 0 & $6,55^{\mathrm{ns}}$ & 4 & - & - & $28,50^{* *}$ & 9 \\
\hline $8^{\mathrm{a}}$ & $0,01^{\mathrm{i}}$ & 0 & $2,36^{\mathrm{ns}}$ & 1 & 0 & - & $16,19^{\text {ns }}$ & 10 \\
\hline $9^{\mathrm{a}}$ & $0,57^{\mathrm{i}}$ & 0 & $10,35^{*}$ & 3 & $73,31^{\mathrm{i}}$ & 0 & $11,68^{*}$ & 5 \\
\hline $10^{\mathrm{a}}$ & $0,18^{\mathrm{i}}$ & 0 & $0,44^{\mathrm{i}}$ & 0 & $1,12^{\mathrm{i}}$ & 0 & $6,28^{*}$ & 2 \\
\hline $11^{\mathrm{a}}$ & $3,88^{*}$ & 1 & $4,17^{\mathrm{ns}}$ & 2 & $3,24^{\mathrm{i}}$ & 0 & $11,39^{* *}$ & 1 \\
\hline $12^{\mathrm{a}}$ & $0,16^{\mathrm{i}}$ & 0 & 0 & - & $52,45^{* *}$ & 3 & $5,97^{*}$ & 1 \\
\hline $13^{\mathrm{a}}$ & $2,40^{\mathrm{ns}}$ & 3 & $1,46^{\mathrm{ns}}$ & 2 & $0,60^{\text {ns }}$ & 1 & $3,77^{\mathrm{ns}}$ & 2 \\
\hline $14^{\mathrm{a}}$ & $13,59^{*}$ & 5 & $0,63^{\mathrm{ns}}$ & 2 & $4,41^{\mathrm{ns}}$ & 5 & $10,50^{\mathrm{ns}}$ & 11 \\
\hline
\end{tabular}

ns - Não significativo, ${ }^{*}$ - significativo ao nível de $5 \%,{ }^{*}$ - significativo ao nível de $1 \%,{ }^{\mathrm{i}}=$ insuficiência de classes, $\mathrm{X}^{2}$ - valor do qui-quadrado calculado, GL - graus de liberdade, nc - número de classes observadas no campo.

DAE quando o índice apresentou-se com valor de 0,9898 indicando regularidade nesta amostragem (Tabela II). Já na cultivar $B t$ todos os valores encontrados estiveram entre 1,7676 e 14, 5687 (Tabela I), indicando também agregação.

Pelos resultados obtidos a partir do índice de Morisita, verificaram-se que em todas as avaliações, nas duas cultivares, os valores foram superiores à unidade com significância estatística de $1 \%$ de probabilidade; apenas aos 82 DAE na cultivar convencional, houve exceção a esta regra, pois nesta amostragem a média de indivíduos de $B$. tabaci nas plantas foi muito baixa e o índice de Morisita resultou em zero.

Desta forma, é possível afirmar que os indivíduos da população amostrada de $B$. tabaci apresentam disposição agregada ou contagiosa no campo, tanto na cultivar $B t$ geneticamente modificada, quanto na cultivar não- $B t$ convencional. Tal resultado talvez não se repita ao se estudar a mesma espécie em outras culturas como ocorreu, por exemplo, na pesquisa de Pereira et al. (2004) que, ao estudar a população desse inseto em feijoeiro, constataram a disposição regular ou uniforme dos indivíduos da população amostrada.

Distribuições teóricas de freqüências: Os testes de ajustes das classes de freqüências observadas em campo dos indivíduos de $B$. tabaci às freqüências calculadas das classes teóricas da Distribuição de Poisson (Tabela III) indicaram que os dados obtidos para este hemíptero não atingiram um ajuste adequado a esse padrão de distribuição. Na cultivar convencional, das 14 amostragens apenas a $5^{\mathrm{a}}$ (46 DAE) e a $12^{\mathrm{a}}$ (82 DAE) não apresentaram número de classes suficientes para fazer a análise de Poisson, porém nenhuma amostragem restante se ajustou a esta distribuição de freqüências, sendo que apenas a $10^{\mathrm{a}}$ (71 DAE) amostragem apresentou diferença significativa de $5 \%$ de probabilidade para as classes observadas, enquanto todas as demais apresentaram diferenças ainda superiores, com significância de $1 \%$.

Também na cultivar Bt, nenhuma das 14 amostragens realizadas mostrou ajuste à distribuição de Poisson, apesar de todas apresentarem um número total de classes suficientes para a realização dos testes. Apenas a $2^{\mathrm{a}}$ (35 DAE) amostragem apresentou significância de $5 \%$ de probabilidade, enquanto as demais resultaram em valores de qui-quadrado com diferenças de ajuste a esta distribuição no nível de $1 \%$ de significância (Tabela III).

Com relação aos testes de ajuste à distribuição binomial negativa do número de indivíduos de $B$. tabaci encontrados no campo amostral (Tabela IV), percebe-se que na cultivar convencional todas as avaliações obtiveram bom ajuste, haja vista que apenas a $5^{\mathrm{a}}$ (46 DAE), $10^{\mathrm{a}}$ (71 DAE) e $12^{\mathrm{a}}$ (82 DAE) amostragens não apresentaram número classes suficientes para se fazer o ajuste a esta distribuição de freqüências, e, das onze amostragens restantes, dez apresentaram ajuste perfeito a esta distribuição, o que pode ser comprovado pela diferença não significativa dos qui-quadrados calculados com os quiquadrados tabelados da distribuição binomial. Uma única exceção ocorreu na $9^{\mathrm{a}}$ (67 DAE) avaliação que apresentou qui-quadrado significativo no nível de $5 \%$ de probabilidade. Mesmo considerando que a primeira e a última avaliação apresentaram baixas médias de indivíduos encontrados, e que a $9^{\text {a }}$ avaliação resultou em diferença significativa a esta distribuição teórica com $95 \%$ de probabilidade, pode-se afirmar que houve ajuste, com elevada confiabilidade, das classes observadas no campo de algodão convencional às classes teóricas desse tipo de distribuição espacial.

$\mathrm{Na}$ cultivar $B t$, todas as 14 avaliações realizadas apresentaram número de classes suficientes para análise da distribuição binomial negativa, sendo que as $1^{\text {a }}$ (28 DAE), $2^{\mathrm{a}}$ (35 DAE), $3^{\mathrm{a}}$ (42 DAE), $6^{\mathrm{a}}$ (59 DAE), $8^{\mathrm{a}}$ (73 DAE), $13^{\mathrm{a}}$ (95 DAE) e $14^{\mathrm{a}}$ (102 DAE) amostragens apresentaram ajuste à distribuição binomial negativa, enquanto outras quatro $\left(4^{\mathrm{a}}, 5^{\mathrm{a}}, 7^{\mathrm{a}}\right.$ e $\left.11^{\mathrm{a}}\right)$ apresentaram resultados que diferem dessa distribuição no nível de $1 \%$ de significância, e três $\left(9^{\mathrm{a}}, 10^{\mathrm{a}}\right.$ e $12^{\mathrm{a}}$ ) mostraram diferença com significância de 5\% de probabilidade para este tipo de arranjo espacial. 
Tabela V. Teste qui-quadrado de aderência de Aphis gossypii e Bemisia tabaci (Binomial Positiva) em algodoeiro DeltaOpal ${ }^{\circledR}$ e DP90B ${ }^{\circledR}$ em Caarapó, MS, 2007.

\begin{tabular}{|c|c|c|c|c|c|c|c|c|}
\hline \multirow[b]{3}{*}{ Amostras } & \multicolumn{4}{|c|}{ DeltaOpal $^{\circledR}$} & \multicolumn{4}{|c|}{ DP90B $^{\circledR}$} \\
\hline & \multicolumn{2}{|c|}{ Aphis gossypii } & \multicolumn{2}{|c|}{ Bemisia tabaci } & \multicolumn{2}{|c|}{ Aphis gossypii } & \multicolumn{2}{|c|}{ Bemisia tabaci } \\
\hline & $\mathrm{X}^{2}$ & GL(nc-3) & $\mathrm{X}^{2}$ & GL(nc-3) & $\mathrm{X}^{2}$ & GL(nc-3) & $\mathrm{X}^{2}$ & GL(nc-3) \\
\hline $1^{\mathrm{a}}$ & 0,01 & - & $3088,84^{\mathrm{i}}$ & 0 & - & - & 202,86 & - \\
\hline $2^{\mathrm{a}}$ & 4,84 & - & 1411,14 & - & 6,14 & - & $1302,06^{\mathrm{i}}$ & 0 \\
\hline $3^{\mathrm{a}}$ & 5,29 & - & 0,09 & - & 0,64 & - & 10,68 & - \\
\hline $4^{\mathrm{a}}$ & 67,24 & - & 1,21 & - & 4,24 & - & 19,36 & - \\
\hline $5^{\mathrm{a}}$ & 0,01 & - & $77,64^{\mathrm{i}}$ & 0 & 0,25 & - & 72,25 & - \\
\hline $6^{\mathrm{a}}$ & 0,01 & - & $214,69^{i}$ & 0 & $614,8^{\mathrm{i}}$ & 0 & 47,61 & - \\
\hline $7^{\mathrm{a}}$ & 0,01 & - & 19,04 & - & - & - & 16 & - \\
\hline $8^{\mathrm{a}}$ & 2,73 & - & 5296,05 & - & 0 & - & 31,36 & - \\
\hline $9^{\mathrm{a}}$ & $1911,75^{\mathrm{i}}$ & 0 & 0,09 & - & 0,04 & - & 2,25 & - \\
\hline $10^{\mathrm{a}}$ & 106,85 & - & $69,52^{\mathrm{i}}$ & 0 & 0.01 & - & $419,87^{\mathrm{i}}$ & 0 \\
\hline $11^{\mathrm{a}}$ & 0,16 & - & $3467,5^{\mathrm{i}}$ & 0 & $268,58^{\mathrm{i}}$ & 0 & $982,38^{\mathrm{i}}$ & 0 \\
\hline $12^{\mathrm{a}}$ & 0,01 & - & $1,04 \mathrm{E}-06$ & - & 323,16 & - & 0,04 & - \\
\hline $13^{\mathrm{a}}$ & 3160,3 & - & $732,71^{\mathrm{i}}$ & 0 & 147,39 & - & 5279,01 & - \\
\hline $14^{\mathrm{a}}$ & 7,84 & - & $3453,94^{\mathrm{i}}$ & 0 & 5,76 & - & 48,76 & - \\
\hline
\end{tabular}

$\mathrm{i}=$ insuficiência de classes, $\mathrm{X}^{2}$ - valor do qui-quadrado calculado, GL - graus de liberdade, nc - número de classes observadas no campo.

Por fim, destaca-se que o teste do qui-quadrado de aderência à distribuição binomial positiva (Tabela V) mostrou que esse hemíptero não apresenta nenhuma tendência para esse tipo de distribuição. Dentre as amostragens realizadas, em ambas cultivares, nenhuma obteve graus de liberdade suficiente para a realização dos testes de ajustes das freqüências observadas em campo às freqüências calculadas dessa distribuição teórica.

O arranjo espacial de $A$. gossypii e $B$. tabaci na cultura do algodoeiro mostrou-se agregado na cultivar $B t \mathrm{em}$, praticamente, todas às vezes que se obteve número de classes suficiente para os testes de ajuste às distribuições teóricas de freqüência. A mesma conclusão foi obtida quando se calculou os índices de agregação, pois todos esses índices também indicaram distribuição tipo agregada para os insetos estudados nessa cultivar de algodoeiro avaliada. No entanto, com relação ao tratamento convencional, apenas B. tabaci ajustou-se totalmente à distribuição do tipo agregada, enquanto que com relação a $A$. gossypii, nenhuma das distribuições teóricas de freqüência ajustou-se completamente aos dados observados no campo.

É possível concluir, portanto, que os adultos de $B$ tabaci não foram influenciados pela cultivar geneticamente modificada, uma vez que, nas duas cultivares, os indivíduos das populações avaliadas apresentaram a mesma forma de distribuição espacial agregada ou contagiosa. No entanto, com relação ao arranjo espacial de $A$. gossypii, é possível que o cultivo $B t$ tenha alguma influência sobre o seu padrão normal de distribuição, pois, ao se analisar os resultados obtidos com as distribuições teóricas de freqüência, percebe-se que esses insetos apresentaram uma clara disposição agregada na cultivar não- $B t$, mas não na cultivar $B t$. Porém, os índices de agregação não evidenciaram essa diferença, apontando a distribuição agregada nas duas cultivares.

Este modelo de distribuição espacial agregada exige um maior número de unidades amostrais do que qualquer outro tipo de distribuição espacial quando da realização de um processo de amostragem. A ausência das lagartas alvo da tecnologia $B t$ parece ter favorecido o crescimento das populações de B. tabaci e $A$. gossypii na cultivar DP90B ${ }^{\circledR}$. Assim, sugere-se que trabalhos futuros busquem desenvolver planos de amostragem seqüencial dessas duas espécies pragas do algodoeiro, tanto no cultivo convencional quanto no cultivo $B t$, visando definir o número exato de unidades amostrais a serem utilizadas. O número preciso de unidades de amostra para o processo de amostragem desses insetos é extremamente importante uma vez que essas espécies são causadoras de consideráveis prejuízos diretos à cultura, além disso, são vetores de muitas viroses causadoras de danos ao algodoeiro.

Assim, quanto ao controle desses insetos pragas, é possível recomendar a aplicação de inseticidas seletivos quando necessário em áreas localizadas da cultura, onde são encontrados grupos destes indivíduos, o que diminui a quantidade de inseticidas pulverizados no meio ambiente, reduzindo os riscos de intoxicação humana, além de permitir maior desenvolvimento dos inimigos naturais das pragas. Sobre esse aspecto, Guedes \& Fragoso (2000) salientam que a aplicação de inseticidas deve ser feita com a menor freqüência possível, limitado à área de ocorrência dos insetos quando possível, mantendo, assim, áreas não-tratadas para fins de refúgio de insetos benéficos; esses pesquisadores recomendam, ainda, a aplicação de inseticidas no estágio mais vulnerável da praga.

Nesses termos, também Metcalf (1980) ressalta que é importante providenciar refúgios para sobrevivência das populações de insetos benéficos nos agroecossistemas. Aplicações localizadas são ecologicamente preferíveis em relação à aplicação em área total e é amplamente praticada em programas de MIP em vários países. Este estudioso cita exemplos da literatura, como no caso do incremento do controle biológico natural de cochonilhas em pomares de citros, na Califórnia (EUA), que tem melhorado grandemente devido à pulverização alternada de ruas em intervalos de seis 
em seis meses, além da pulverização em faixas de ruas de culturas anuais, como o algodoeiro, o que pode proporcionar similares refúgios para inimigos naturais.

\section{REFERÊNCIAS}

Araújo, A. E. \& N. D. Suassuna. 2003. Guia de identificação e controle das principais doenças do algodoeiro no estado de Goiás. (Documentos, 113). Campina Grande, EMBRAPA-CNPA, 40 p.

Barbosa, J. C. 1992. A amostragem seqüencial, p. 205-211. In: Fernandes, O. A., Correia, A. C. B., de Bortoli, S. A. (ed.). Manejo integrado de pragas e nematóides. Jaboticabal, FUNEP, xii +253 p.

Chu, C. C.; T. J. Henneberry \& A. C. Cohen, 1995. Bemisia argentifolii (Homoptera: Aleyrodidae) on cotton: host preference and factors affecting oviposition and feeding site preference. Environmental Entomology 24: 354-360.

Chu, C. C.; T. P. Freeman; J. S. Buckner; T. J. Henneberry; D. R. Nelson \& E. Natwick. 2001. Susceptibility of upland cotton cultivars to Bemisia tabaci biotype B (Homoptera: Aleyrodidae) in relation to leaf age and trichome density. Annals of the Entomological Society of America 94: $743-749$.

Degrande, P. E. 1998. Guia prático de controle das pragas do algodoeiro. Dourados, Universidade Federal do Mato Grosso do Sul, 60 p.

Elliot, J. M. 1979. Some methods for the statistical analysis of sample of benthic invertebrates. Sci. Publ. Freshwater Biological Association 25, $148 \mathrm{p}$.

Fernandes, M. G.; A. C Busoli \& J. C. Barbosa. 2003. Distribuição espacial de Alabama argillacea (Hübner) (Lepidoptera: Noctuidae) em algodoeiro, Neotropical Entomology 32: 117-122.

Guedes, R. N. C. \& D. B. Fragoso. 2000. Insetos com vontade de viver, Cultivar Hortaliças e Frutas 4: 18-20.

Kuno, E. 1991. Sampling and analysis of insect populations. Annual Review of Entomology 36: 285-304.

Liu, X. D.; B. P. Zhai; X. X. Zhang \& J. M. Zong. 2005. Impact of transgenic cotton plants on a non-target pest, Aphis gossypii Glover. Ecological Entomology 30: 307-315.

Metcalf, R. L. 1980. Changing role of insecticides in crop protection. Annual Review of Entomology 2: 219-256.

Michelotto, M. D. \& A. C. Busoli. 2007. Caracterização da transmissão do vírus do mosaico-das-nervuras do algodoeiro pelo pulgão Aphis gossypii com relação à persistência e ao tempo necessário para inoculação. Bragantia 66: 441-447.

Naranjo, S. E. \& H. M. Flint. 1995. Spatial distribution of adult Bemisia tabaci (Homoptera: Aleyrodidae) in cotton and development and validation of fixedprecision sampling plans for estimating population density. Environmental Entomology 24: 261-270.

Pereira, M. F. A.; A. L. Boiça Jr. \& J. C. Barbosa, 2004. Spatial distribution of Bemisia tabaci (Genn.) biotype B (Hemiptera: Aleyrodidae) on bean crop (Phaseolus vulgaris L.). Neotropical Entomology 33: 499-504.

Poole, R. W. 1974. An introduction to quantitative ecology. New York, McGraw Hill, 525 p.

Rabinovich, J. E. 1980. Introducción a la ecología de poblaciones animales. México, Continental, 313 p.

Ricklefs, R. E. 2003. A economia da natureza, $5^{\text {a }}$ ed. Rio de Janeiro, Guanabara/Koogan. 470 p.

Santos, W. J. 2001. Identificação, biologia, amostragem e controle das pragas do algodoeiro, p. 181-226. In: Algodão: Tecnologia de produção. Dourados, EMBRAPA Agropecuária Oeste \& EMBRAPA Algodão. 296 p.

Silveira Neto, S.; O. Nakano; D. Barbin \& N. A. Villa Nova, 1976. Manual de ecologia dos insetos. São Paulo: Agronômica Ceres. 419 p.

Southwood, T. R. E. 1978. Ecological methods, with particular reference to the study of insect populations. 2 ed. London, Chapman and Hall. $524 \mathrm{p}$.

Young, L. J. \& J. H. Young. 1998. Statistical ecology: a population perspective. Boston, Kluwer Academic Publishers, 565 p. 